\title{
A POL YNOMIAL FUNCTION IN THE AUTOMATIC RECONSTRUCTION OF FRAGMENTED OBJECTS
}

\author{
Nada A. Rasheed and Md Jan Nordin \\ Faculty of Information Science and Technology, \\ Universiti Kebangsaan Malaysia, UKM, Bangi, Malaysia
}

Received 2014-04-11; Revised 2014-09-03; Accepted 2014-11-29

\begin{abstract}
The reconstruction of archaeological objects is a very challenging problem and solving this problem is important. Occasionally, archaeological workers suffer when trying to match object fragments together, especially when there is a presence of significant gaps in the fragments, or even in the case of similarity, where fragments are mixed with fragments of other objects. The main theme of this study is a proposed method for the reconstruction of pottery from archaeological fragmented pots and vases, depending on the use of a polynomial function. In any case, there is an important fact that should be mentioned: The assembly of any object will rely on the edges of the fragment firstly, then the color and texture. Therefore, this study has adopted the edges of the fragments as a condition when reconstructing the objects, by exploiting the edges of the fragments as an important feature, mainly due to the fact that edges of the fragments are lines, corners and curves. A Canny filter was used to identify the edges of the fragments. In addition, for the purpose of obtaining the vector of coefficient for the set of edges, a polynomial function algorithm was applied. Lastly, the experiments shows that the algorithm is effective, especially when applying the correlation coefficient formula in the classification phase by using the data set which consists of 56 pieces and each one has edges at a rate of 3-5 $\mathrm{cm}$. The experimental results achieved a high success rate that means the proposed system may produce high performance to recognize and match the edges by using a polynomial function to extract features and to classify them by using a correlation coefficient.
\end{abstract}

Keywords: Assembly Shards, Pottery, Fragment Matching, Polynomial Function, Edges

\section{INTRODUCTION}

One of the major unresolved and difficult problems that occur through the field of computer vision is to reassemble broken or torn objects, such as archeological objects, documents, paleontology and art conservation, especially when exploring archaeological objects that are frequently in the case of being broken. Therefore, it is of great interest that the objects are assembled before they are lost or damaged (Castañeda et al., 2011; Oxholm and Nishin,
2012). Another important issue is finding solutions that challenge the accurate restoration of the archaeological fragments to their original form because of their high value to scholars and their heritage, that represents the past civilizations and cultures and helps archaeologists make inferences about past civilizations (Funkhouser et al., 2011). As mentioned earlier, the artifacts are often found in a fractured state and the process of manually reconstructing them is time consuming and may take years of tedious work (Son et al., 2013). Therefore, their reconstruction is a challenging task, especially if they Corresponding Author: Nada A. Rasheed, Faculty of Information Science and Technology, Universiti Kebangsaan Malaysia, UKM, Bangi, Malaysia 
exceed thousands of fragments and the assembly process seems to be similar to assembling a jigsaw puzzle (Castañeda et al., 2011; Funkhouser et al., 2011).

Recently, while the world has been witnessing a development, especially in the performance of computers as the use of image processing and pattern recognition techniques have emerged, many of the authors found algorithms to assist them in solving many problems. Thus, this study aims to find a solution for assembling archaeological fragments depending on the edges of the fragment to achieve high accuracy with the least time. To highlight the technique used in this study, the main feature to achieve accurate matching between a pair of fragments is to use the edge curve, because each edge is a curve that contains angles and curvatures. Therefore, it will be applied to the polynomial function to calculate the function for each edge and by comparing the coefficients of functions for both edges, the classification will be done based on the values of the coefficients and their signs. This study is structured as follows: The present background of study is drawn in section 2. An overview of the polynomial function is presented in section 3. Section 4 describes the system overview. Section 5 gives the details of experiments and results. Finally, a conclusion is given in section 6 .

\section{RELATED WORKS}

For the purpose of utilization of historical monuments and protecting them from damage, which they may be exposed to due to negligence or attempts to assemble them manually, it is necessary to find automatic solutions by using computer techniques. So much work has been done on the reassembling of fragments in both 2 and 3-dimensions. In order to highlight the most important studies that have addressed this problem based on 2-dimensional techniques, several important studies are taken into consideration. Leitao and Stolfi (2005) provided many of the studies in the field of assembling pottery fragments and the latest was in 2005 . This study determined pottery fragments that were matched by measuring the average amount of information contained in the form of a break line of a given length. Subsequently, most of the previous work focused on finding pairwise matches between adjacent fragments using surface color and texture, without reference to the use of the fragment edges and this is found in (Smith et al., 2010), where they suggested methods for ceramic fragments that were highly textured and classified them based on the color and texture characteristics. Generally, their algorithm resulted in better accuracy in the case of assembling the fragments and relying on their characteristics of color and texture. On the other hand, others relied on texture features only for classification of ancient-ceramic fragments, such as Ying and Gang (2010); their method can't be applied when fragments do not have a texture.

Toler-Franklin et al. (2010) disagreed with the authors who relied on the traditional features in the classification of archaeological fragments and they were relying on multiple-features that were extracted from fresco fragments based on color, shape and normal maps. Another work by (Kimia and Aras, 2010), includes a framework, or a practical system, that can be used by archaeologists in reassembling 2 dimensional archeological vessel fragments and that could be applied to 3dimensional fragments. Zhang et al. (2011) focused on the contour of the fragment in the matching process and their proposed method relies on a curve matching algorithm that is based on the multiscale space. On the other hand, a variety of algorithms have been proposed to reconstruct the fragments of wall panels excavating of the Greek island Thera (Santorini), which were painted in 1600 B.C. Skembris et al. (2012) proposed a methodology for the reconstruction of 2-dimensional fragmented wall paintings based on the maximum possible information extracted from the shape (contour) characteristics of the pieces, the chromatic and thematic content.

Another type of study on classification of fragments was proposed by (Makridis and Daras, 2012), they focused on the features that are extracted based on chromaticity and chrominance (color) and the low level features, such as standard deviation and the contrast. Also the medium level features such as the extraction of the edges of the fragment are carried out by using a kirsch edge map and extracting the texture depending on the LBP method. The results they obtained after testing the model on the pottery database with a total of 62 fragments achieved a success rate $70.97 \%$, while the results after testing the model on the ceramic database of a total of 46 fragments, it achieved a success rate $78.26 \%$. Also, to reconstruct fresco fragments, Funkhouser et al. (2011) investigated a machine learning method which computes the probability of the matching correctness, combining multiple effective features.

\section{POLYNOMIAL FUNCTION}

A polynomial function is a function such as a quadratic, a cubic, among others, involving only nonnegative integer powers of $(\mathrm{x})$. The general form is: 


$$
f(x)=a_{n} x^{n}+a_{n-1} x^{n-1}+\ldots \ldots . .+a_{2} x^{2}+a_{1} x+a_{0}
$$

where, $(x)$ is a variable and $\left(a_{n}, a_{n-1}, \ldots \ldots, a_{2}, a_{1}, a_{0}\right)$ are given constants which are real numbers called the coefficients of the polynomial. Also, $(n)$ is the degree of the formula and must be a positive integer, i.e., $a_{n} \neq 0$ (Sturmfels, 2002).

\section{SYSTEM OVERVIEW}

The proposed technique consists of several sets of procedures; each one performs a specific job that can be summarized into two stages. The first stage includes image acquisition by the camera and the next procedure is preprocessing, which is done to improve the robustness of the features that will be extracted. This requires some manipulation before the application of any classification technique. To reduce the computational effort during puzzling, particularly important with large amounts of data, the edges filtering procedure is carried out. The second stage includes applying the polynomial function to get the coefficients with their signs. Finally, the classification process is conducted within each group based on the values of the coefficients and their signs. The proposed system consists of the following steps, as depicted in Fig. 1.

\subsection{Data Acquisition and Processing}

In order to apply the proposed algorithm, the images of the fragment must be obtained by capturing them via camera Nikon with a resolution of 24 MP. It will be captured each edge of the fragment with the image size is $6000 \times 4000$ pixels. Additionally, careful consideration of reflections and shadows is taken, which are generated by the camera flash. This study aims to reconstruct fragments of 3 pots consisting of 56 pieces of pottery fragments that were obtained in cases, Fig. 2.

The fragments are mixed and irregular, with different sizes ranging between $7-30 \mathrm{~cm}$ in width and $9-35 \mathrm{~cm}$ in length. In order to eliminate irrelevant information and to enhance the robustness of features to be extracted, the images require some manipulation before the application of any classification technique. The preprocessing is performed in order to reduce noise in the input images, where the original image may contain noisy objects due to the camera flash, or for other reasons such as the variability of the lighting. Therefore, this issue is solved by the use of Gaussian Filter, because it is widely used for noise reduction and that is expressed in (Imajo, 2012).

\subsection{Edge Detection}

Incidentally, it should be mentioned that the pottery fragments include pots, vases, bowls, water jugs, wall paintings and so on, where the edges of the fragments vary from one object to another. Sometimes they are thick, such as frescoes and other times they are narrow and sharp, such as vases and vessels. Therefore, this study attempts the reconstruction all kinds of pottery edges. The task of the feature extraction process is obtaining the most relevant information from the original data and representing a lower dimensional space that helps to identify the subset of features by preserving only the most important and filtering or excluding all others.

Thus, this study relies on the edges extracted from an image, which is an important feature in the next phase. To obtain the edges of the fragments accurately, the Canny filter algorithm is applied, following a method used in (Ding and Goshtasby, 2001), which has achieved excellent results in the extraction of edges. For instance, the results of using this algorithm are shown in Fig. 3.

In this manner, all of the edge points are stored in an array which is assigned the specific coordinates of each point related to the edge fragments only, so it is obtained as a set of points and each one has coordinates $(x, y)$ :

$$
C=\left\{P\left(x_{i}, y_{i}\right), \forall\left(x_{i}, y_{i}\right)=1\right\}
$$

where, $i=1 \ldots n, j=1 \ldots m$

On the basis of these couples of pixels $\mathrm{P}\left(x_{i}, y_{j}\right)$, the curve is plotted according the values of $\mathrm{x}$ on the $\mathrm{x}$-axis and the corresponding values for the $x$-values on the $y$ axis, as shown in Fig. 4.

\subsection{Apply the Polynomial Function}

In order to highlight the finding of the polynomial equations, there are several strategies that help to find the coefficients of polynomial equations for each object. The one strategy that through experience was frequent in the matching of fragments, the high amount of error when the curve was a vicious circle was noticed, hence, it was decided to split pixels of the curve into two equal lengths. Then each set will be:

$$
C=\left\{P\left(x_{i}, y_{j}\right) / 2\right\}
$$

where, $i=1 \ldots n / 2, j=1 \ldots m / 2$

It was found that the curved points dispersed and were divergent with each other and this generates residuals when obtaining the polynomial equation. 


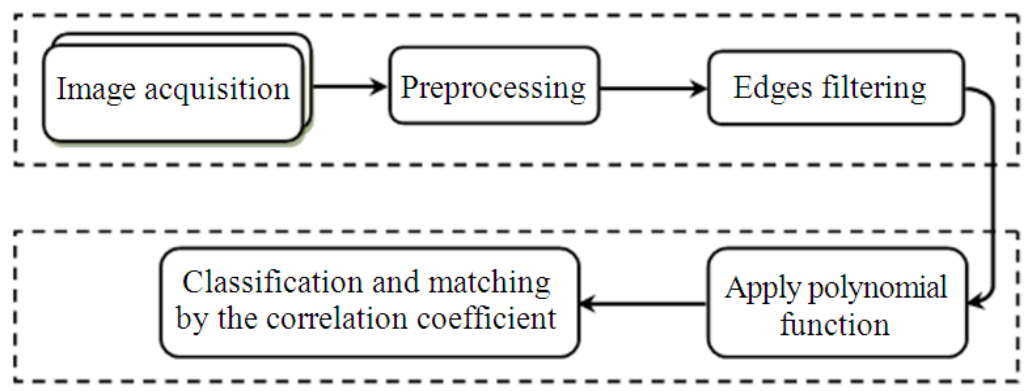

Fig. 1. Diagram of proposed system

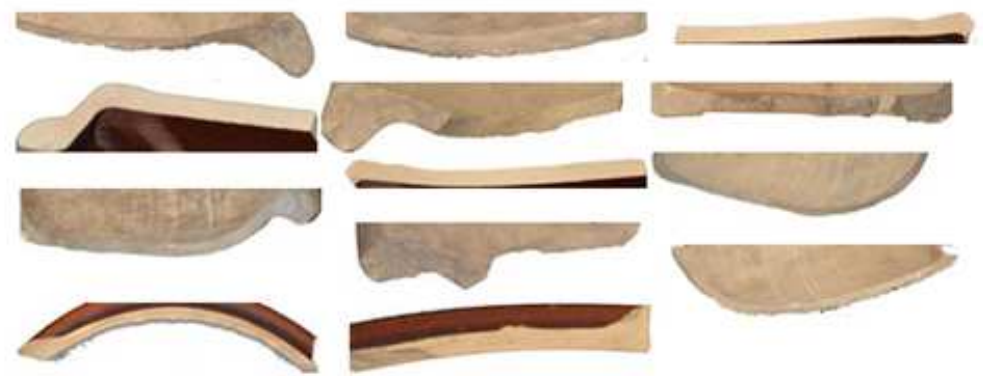

Fig. 2. Sample of data sets that used to apply the algorithm
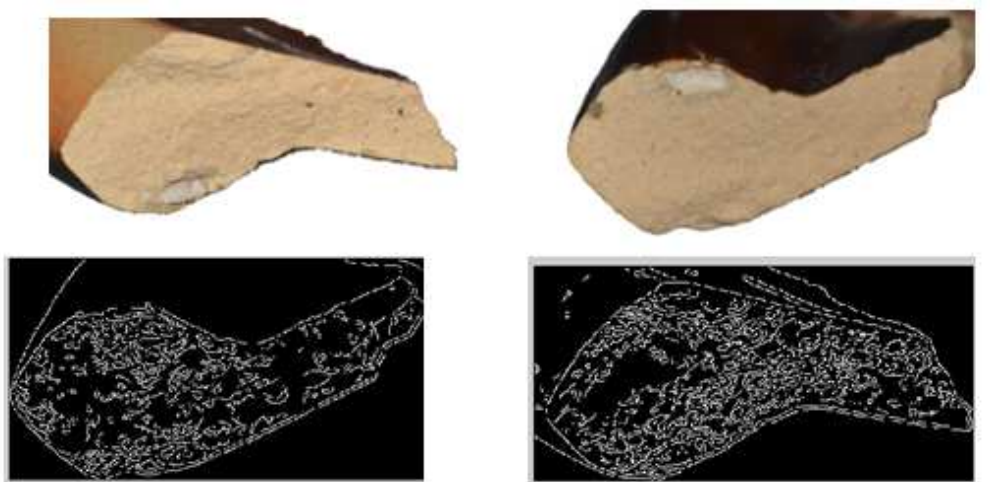

Fig. 3. Edges extraction using the canny algorithm

Therefore by applying another strategy using the standard score Equation 2 as shown below improved the points properties and reduced the distractions to approximate the points of the curve:

$$
z=\frac{x_{i}-\mu}{\sigma}
$$

where, $x_{i}$ represents the original point value, $\mu$ and $\sigma$ represent the means and standard deviations respectively.
Later, it was desired to find a polynomial equation such that a given number of original points lie on the curve and this reduces the residuals in a model by fitting a higher degree polynomial. Therefore, it will be focusing on nonlinear morphing transformations up to the 6th order degree of nonlinearity that are well approximated and it gives the best results to represent the original curve. This concept is further illustrated in Fig. 5. 

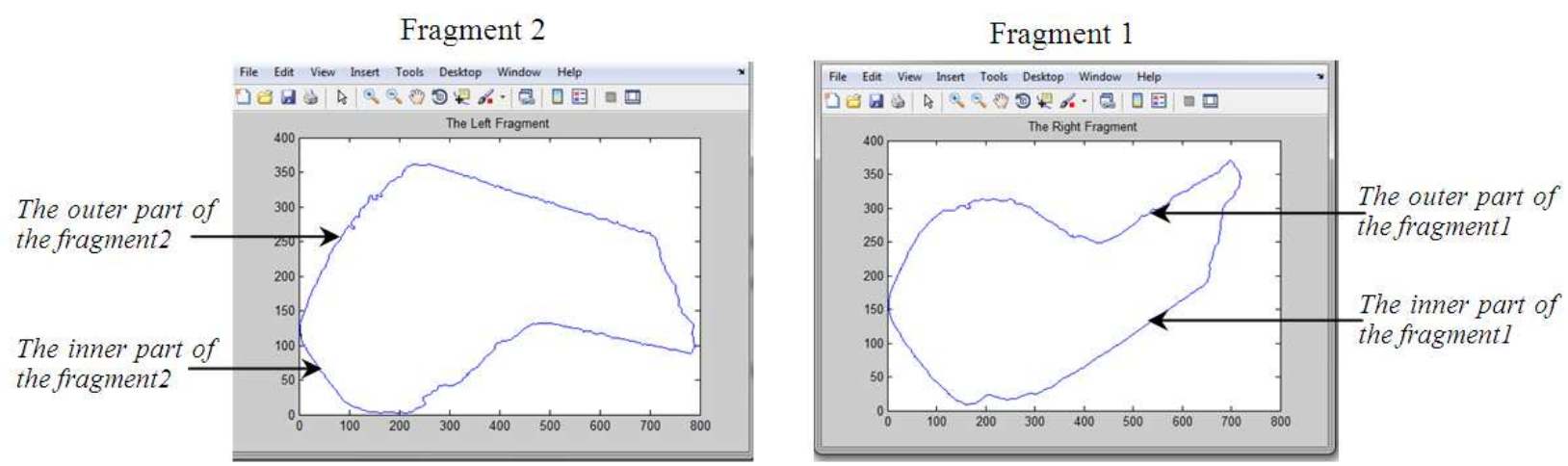

Fig. 4. Plotting the curve according to the set of point
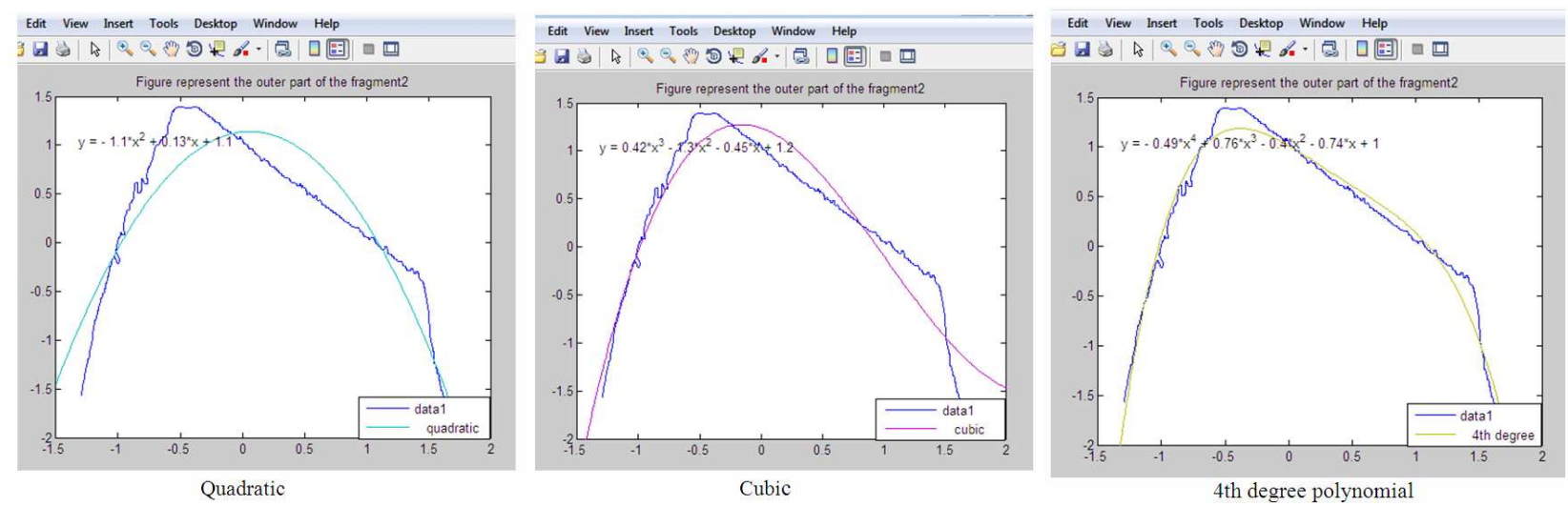

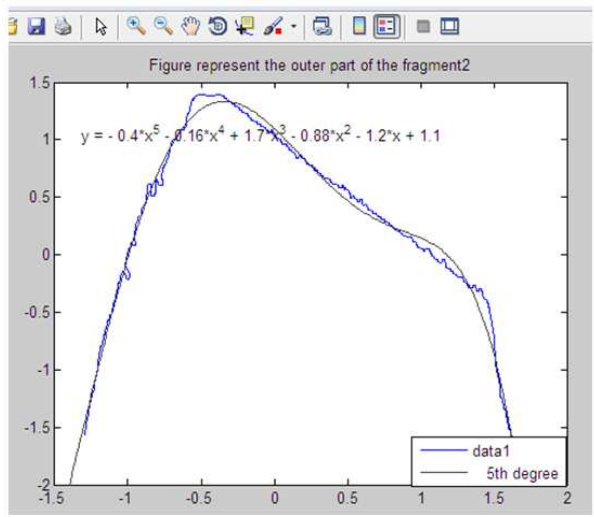

5 th degree polynomial

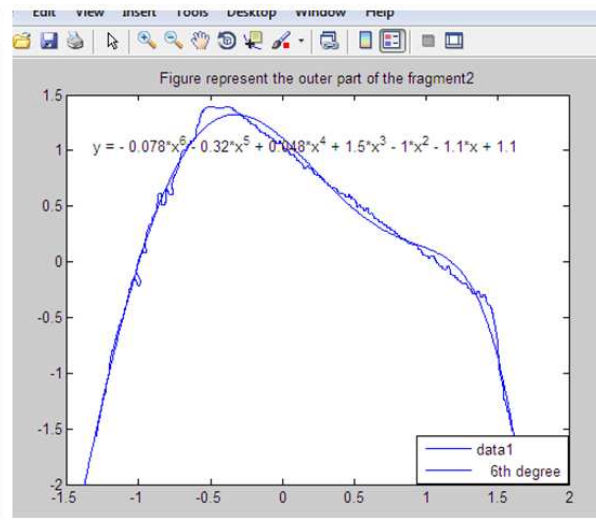

6th degree polynomial

Fig. 5. Polynomial of different degrees

After generating a matrix of $\left(x_{i}, y_{i}\right)$ points for each edge of the fragments, the polynomial function of degree $n$ was evaluated according to those points. As a result, a vector of estimated coefficients of length $n+1$ was returned containing the polynomial coefficients in descending powers for each nonlinear regression of the responses $y_{i}$ according to the $x_{i}$, using the model specified in
Equation 1. The coefficients in the approximating polynomial of the 6th degree are shown in Table 1.

Algorithm procedure for obtain coefficients

Step1: Input edge-fragment

Step2: Calculate the length 
Step3: For $i=1$ to $k$

For $j=1$ to $k$

Calculate the value for $x_{i}, y_{j}$ to each point for the edge of the fragment

end for

Step4: Plot $\left(x_{i}, y_{j}\right)$

Step5: Calculate the Polynomial functions in all points $\left(x_{i}, y_{j}\right)$

$$
f(x)=a_{n} x^{n}+a_{n-1} x^{n-1}+\ldots \ldots .+a_{2} x^{2}+a_{1} x+a_{0}
$$

Step5: Obtain the coefficients edges Fig. 6.

\subsection{Classification using Correlation Coefficient}

This section describes a fragment matching based on the edges of the curve feature. After computing all of the coefficients to all the available edges, the fragments are then clustered by using a correlation coefficient because it produces better classification performance. The correlation coefficients the appointment of the nature and
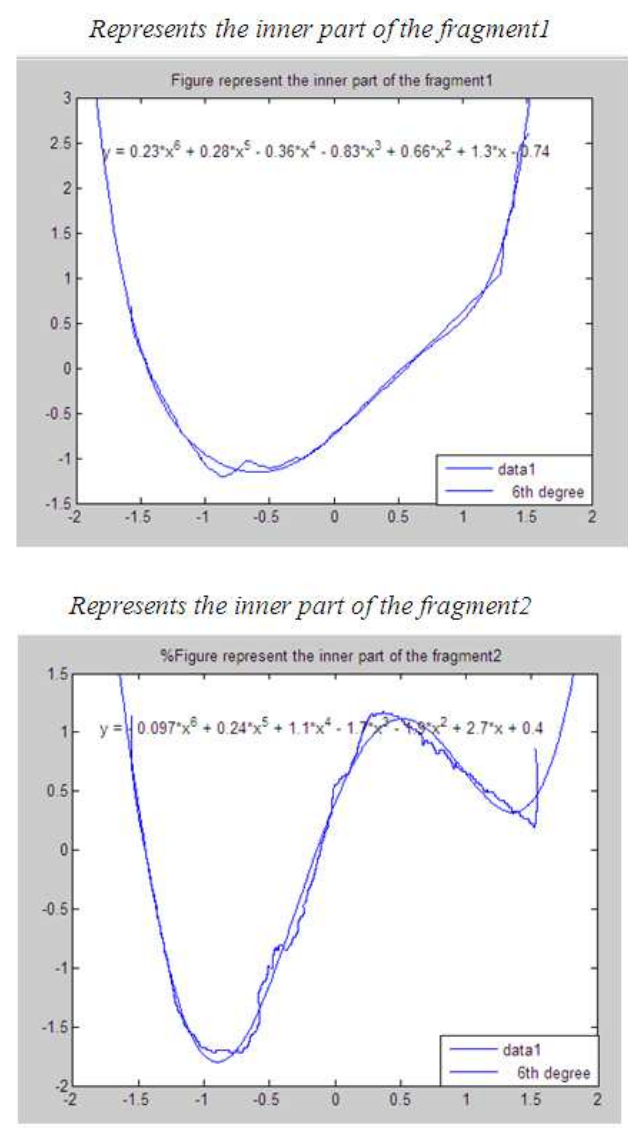

strength of the relationship between two variables, where its value ranges between the $(-1 \leq r \leq+1)$ and the following formula represents the correlation coefficient between two variables, which are $x$ and $y$ in Equation 3 and 4:

$$
r_{x y}=\frac{\mathrm{n} \sum_{i=1}^{n} \mathrm{x}_{\mathrm{i}} y_{i}-\sum_{i=1}^{n} \mathrm{x}_{\mathrm{i}} \sum_{i=1}^{n} y_{i}}{\sqrt{n \sum_{i=1}^{n} \mathrm{x}_{\mathrm{i}}^{2}-\left(\sum_{i=1}^{n} \mathrm{x}_{\mathrm{i}}\right)^{2}} \sqrt{n \sum_{i=1}^{n} \mathrm{y}_{\mathrm{i}}^{2}-\left(\sum_{i=1}^{n} \mathrm{y}_{\mathrm{i}}\right)^{2}}}
$$

Or:

$$
r_{x y}=\frac{\sum_{i=1}^{n} \mathrm{x}_{\mathrm{i}} y_{i}-n \overline{x y}}{\sqrt{\sum_{i=1}^{n} \mathrm{x}_{\mathrm{i}}^{2}-\mathrm{nx}^{2}} \sqrt{\sum_{i=1}^{n} \mathrm{y}_{\mathrm{i}}^{2}-n \bar{y}^{2}}}
$$

Represents the outer part of the fragment1

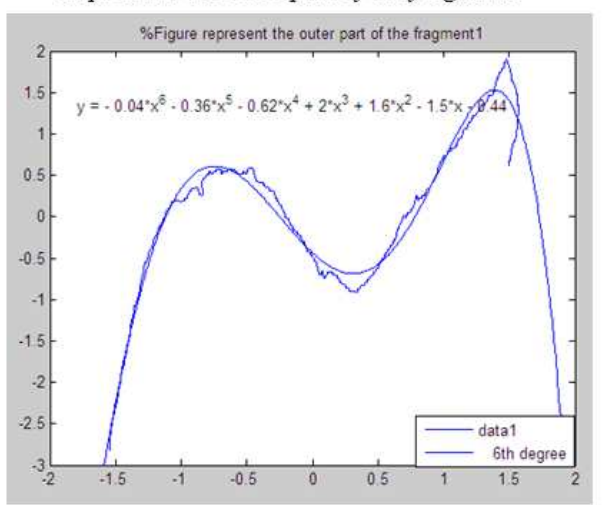

Represents the outer part of the fragment 2

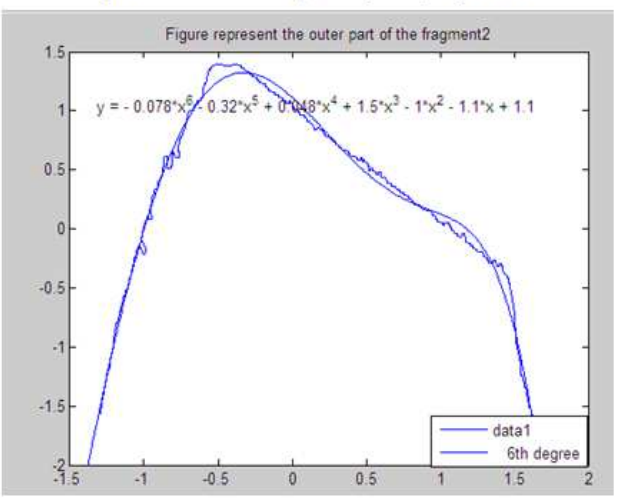

Fig. 6. Applying a polynomial function 


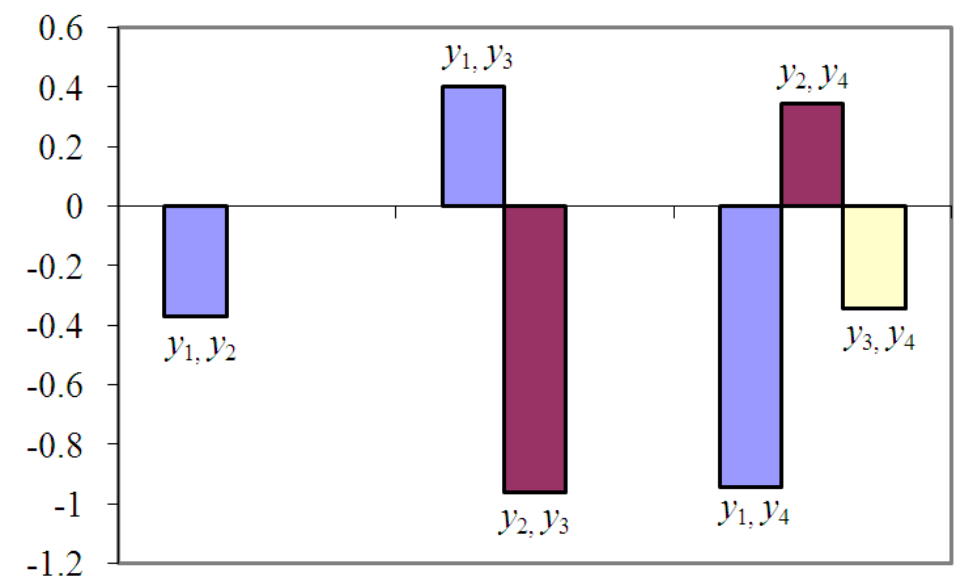

Fig. 7. The results of correlation coefficient

Table 1. The coefficients of function $y$

\begin{tabular}{lllll}
\hline Coefficient & $y_{1}$ & $y_{2}$ & $y_{3}$ & $y_{4}$ \\
\hline$a_{6}$ & 0.23 & -0.04 & -0.097 & -0.08 \\
$a_{5}$ & 0.28 & -0.36 & 0.24 & -0.32 \\
$a_{4}$ & -0.36 & -0.62 & 1.06 & 0.05 \\
$a_{3}$ & -0.83 & 2.02 & -1.71 & 1.51 \\
$a_{2}$ & 0.66 & 1.60 & -1.91 & -1.02 \\
$a_{1}$ & 1.29 & -1.48 & 2.67 & -1.13 \\
$a_{0}$ & -0.74 & -0.44 & 0.39 & 1.11 \\
\hline
\end{tabular}

Table 2. Obtained results by the correlation coefficient

\begin{tabular}{llll}
\hline $\mathrm{r}$ & $y_{2}$ & $y_{3}$ & $y_{4}$ \\
\hline$y_{1}$ & -0.3689 & 0.4005 & -0.9423 \\
$y_{2}$ & & -0.9602 & 0.3451 \\
$y_{3}$ & & & -0.3430 \\
\hline
\end{tabular}

where, $r_{x y}$ is the correlation between the input features $x$ and $y$ and $n$ is the number of coefficients in each polynomial equation.

The aim of this study is to obtain a strong relationship between a pairwise of fragments depending on the coefficient features and identify whether it is positive or negative in order to obtain accurate classification. At the final stage, the correlation coefficient algorithm is applied to obtain the relationship between two sets of coefficients and the mean is calculated $r_{y_{1} y_{2}}, \ldots \ldots ., r_{y_{3} y_{4}}$, the results are shown in Table 2 and Fig. 7.

\section{EXPERIMENTS AND RESULTS}

This section is devoted to presenting the test results for selecting the best matching between a pairwise of pottery fragments according the relationship of their coefficients. By comparing the results of the different correlation coefficient values across the Table 2 and the graph shown in Fig. 7, it was found that there was a negative relationship between the two variables $r_{y_{1} y_{4}}, r_{y_{2} y_{3}}$ and this corresponds to -0.9423 and -0.9602 respectively, which means height matching between the four equations $\left(y_{1}, y_{4}\right)$ and $\left(y_{2}, y_{3}\right)$, as shown in the Equations 5-8 below:

$$
\begin{aligned}
& y_{1}=0.23 x_{1}^{6}+0.28 x_{2}^{5}-0.36 x_{3}^{4} \\
& -0.83 x_{4}^{3}+0.66 x_{5}^{2}+1.29 x_{6}-0.74
\end{aligned}
$$

$$
\begin{aligned}
& y_{2}=-0.04 x_{1}^{6}-0.36 x_{2}^{5}-0.62 x_{3}^{4} \\
& +2.02 x_{4}^{3}+1.60 x_{5}^{2}-1.48 x_{6}-0.44 \\
& y_{3}=-0.97 x_{1}^{6}+0.24 x_{2}^{5}+1.06 x_{3}^{4} \\
& -1.71 x_{4}^{3}-1.91 x_{5}^{2}+2.67 x_{6}+0.39 \\
& y_{4}=-0.08 x_{1}^{6}-0.32 x_{2}^{5}+0.05 x_{3}^{4} \\
& +1.51 x_{4}^{3}-1.02 x_{5}^{2}-1.13 x_{6}+1.11
\end{aligned}
$$

From this result, it can be deduced that the match has been achieved between a couple of the fragments (the inner part of the fragment 1, the outer part of the fragment 2), (the inner part of the fragment 2, the outer part of the fragment 1) and the success rate of this experiment was $100 \%$, Fig. 8. In a broader scope, a number of experiments have been conducted by the proposed method in an effort to recognize and match effectively and achieve the highest ranking for the assembly of the fragments.

As shown in the Table 3 and $\mathbf{4}$ sometimes notice a strong correlation between two edges of the fragments and at the same time one of edges associated with the other edge of another fragment, but a lesser degree. 


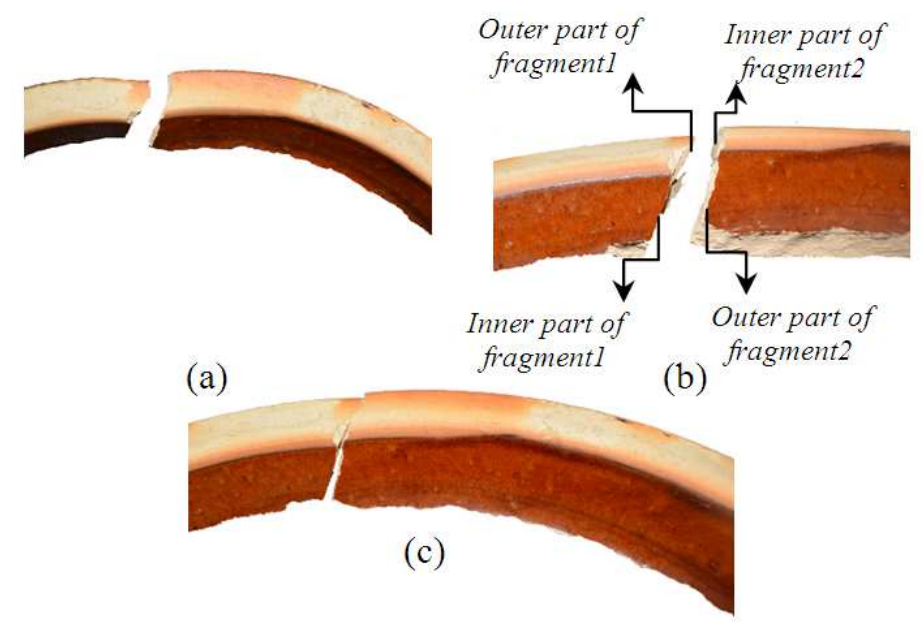

Fig. 8. (a), (b), (c) Represents reconstruction pair was fragmented

Table 3. Coefficient of function $y$ for the sample of data set

\begin{tabular}{|c|c|c|c|c|c|c|c|c|c|c|c|c|}
\hline Coefficient & $\mathrm{y}_{1}$ & $y_{2}$ & $y_{3}$ & $y_{4}$ & $\mathrm{y}_{5}$ & $y_{6}$ & $y_{7}$ & $y_{8}$ & & & $y$ & $y$ \\
\hline$a_{6}$ & -0.056 & -0.46 & 0.516 & -0.015 & 0.031 & -0.021 & 0.3428 & 0.1921 & .. & .. & 1.1911 & -0.1825 \\
\hline$a_{5}$ & -0.287 & -0.309 & -0.088 & -0.111 & -0.089 & -0.207 & -1.2991 & 0.2901 &.. & .. & -0.3065 & 1.271 \\
\hline$a_{4}$ & 0.255 & 2.276 & 0.71 & -0.183 & 0.05 & -0.597 & 0.3973 & -1.1749 &.. & .. & -0.0598 & 0.6614 \\
\hline$a_{3}$ & 1.366 & 1.208 & 0.508 & 0.046 & -0.094 & -0.19 & 2.2608 & -1.1583 &.. & .. & 1.0531 & 0.8317 \\
\hline$a_{2}$ & -0.453 & -3.236 & -0.384 & -0.12 & 0.215 & 0.001 & -0.1217 & 2.4379 &.. &.. & 0.1549 & -0.7502 \\
\hline$a_{1}$ & -1.859 & -0.251 & -1.755 & 1.578 & 0.049 & -0.379 & -2.3732 & 0.8344 & .. & . & 1.1773 & 1.4832 \\
\hline$a_{0}$ & 0.047 & 0.919 & -0.303 & 0.334 & 0.002 & -0.236 & -0.6465 & -0.7734 &.. &.. & -0.2079 & 0.2455 \\
\hline
\end{tabular}

Table 4. The results of the correlation coefficient

\begin{tabular}{|c|c|c|c|c|c|c|c|c|c|c|c|c|c|}
\hline $\mathrm{r}$ & $y_{2}$ & $y_{3}$ & $y_{4}$ & $y_{5}$ & $y_{6}$ & $y_{7}$ & $y_{8}$ & $y_{9}$ & $y_{10}$ & $y_{11}$ & & $y$ & $y$ \\
\hline$y_{1}$ & 0.431 & 0.86 & -0.72 & -0.43 & 0.09 & 0.92 & -0.61 & -0.81 & 0.664 & -0.67 & $\ldots$ & -0.102 & -0.208 \\
\hline$y_{2}$ & & 0.389 & -0.01 & -0.64 & -0.7 & 0.28 & -0.96 & -0.64 & -0.31 & 0.373 & $\ldots$ & -0.051 & 0.529 \\
\hline$y_{3}$ & & & -0.87 & -0.26 & 0.04 & 0.81 & -0.53 & -0.72 & 0.439 & -0.52 & $\ldots$ & -0.17 & -0.323 \\
\hline$y_{4}$ & & & & 0.032 & -0.3 & -0.63 & 0.155 & 0.551 & -0.55 & 0.7 & $\ldots$ & 0.466 & 0.509 \\
\hline$y_{5}$ & & & & & 0.19 & -0.19 & 0.717 & 0.485 & 0.082 & -0.11 & $\ldots$ & -0.041 & -0.676 \\
\hline$y_{6}$ & & & & & & 0.17 & 0.547 & 0.125 & 0.569 & -0.67 & $\ldots$ & 0.208 & -0.614 \\
\hline$y_{7}$ & & & & & & & -0.44 & -0.72 & 0.772 & -0.72 & $\ldots$ & 0.184 & -0.348 \\
\hline$y_{8}$ & & & & & & & & 0.79 & 0.125 & -0.16 & $\ldots$ & 0.044 & -0.408 \\
\hline$y_{9}$ & & & & & & & & & -0.37 & 0.401 & $\ldots$ & 0.28 & 0.096 \\
\hline$y_{10}$ & & & & & & & & & & -0.96 & $\ldots$ & 0.104 & -0.506 \\
\hline$y_{11}$ & & & & & & & & & & & & 0.068 & 0.632 \\
\hline ..... & & & & & & & & & & & & & \\
\hline$y$ & & & & & & & & & & & & & 0.042 \\
\hline
\end{tabular}

Because of the similarity of the edges, so preference will be for the highest degree. For example of this case the relationship between $\left(y_{1}, y_{7}\right)$ equal to $(0.92)$ and the relationship between $\left(y_{1}, y_{3}\right)$ equal (0.86). Occasionally some of the edges have not relationship with others, therefore in this situation another feature will use to reconstruct the fragments. This experience has achieved a success rate in recognizing fragments correctly with accurate matches a percentage amounts $94.2 \%$.
The remaining percentage $5.8 \%$, shows that the system became confused with some recognition. When comparing this study with previous studies, we found that it's an easy way to reconstruct and matching the fragments. Because it depends on the edges of the fragments, where basically when assembling any broken object will be relying on the edges feature and give it priority in the assembly.

When we compare the results that obtained by applying the Polynomial function with the results 
Makridis and Daras (2012), It is clear that the proposed method has achieved better results.

\section{CONCLUSION}

In a nutshell, this study proposed a method for solving the practical problem of reassembling fragments of ancient pottery. The main principle of the proposed method is to extract the edges of the fragments, mainly due to the fact that edges of the fragments are lines, corners and curves. The important points from the edge of the fragment were extracted, then apply the polynomial function to calculate the coefficients of formula depending on the $\mathrm{P}(x i, y j)$ of the curve and through conducting several experiments, estimate coefficients of polynomial formula in the 6th degree were found in the phases of classification and matching. Furthermore, these seven coefficients for each polynomial equation have achieved great results in the process of classification and matching. Incidentally, it is worth noting that this approach is appropriate when the pair of adjacent fragments share a fracture line.

In the future, more details about the features such as the color of pixels may be gives better results with the Polynomial function.

\section{ACKNOWLEDGEMENT}

The researchers wish to thank Universiti Kebangasaan Malaysia (UKM) and Ministry of Higher Education Malaysia for supporting this study by research grant ERGS/1/2012/STG07/UKM/02/9.

\section{REFERENCES}

Castañeda, A.G., B. Brown, S. Rusinkiewicz, T. Funkhouser and T. Weyrich, 2011. Global consistency in the automatic assembly of fragmented artefacts. Proceedings of the 12th International conference on Virtual Reality, Archaeology and Cultural Heritage, (ACH' 11), Switzerland, pp: 73-80. DOI: 10.2312/VAST/VAST11/073-080

Ding, L. and A. Goshtasby, 2001. On the canny edge detector. J. Patt. Recognit., 34: 721-725. DOI: 10.1016/S0031-3203(00)00023-6
Funkhouser, T., H. Shin, C. Toler-Franklin, A.G. Castañeda and B. Brown et al., 2011. Learning how to match fresco fragments. J. Comput. Cultural Heritage, 4: 2-5. DOI: $10.1145 / 2037820.2037824$

Imajo, K., 2012. Fast gaussian filtering algorithm using splines. Proceedings of the 21st International Conference on Pattern Recognition, Nov. 11-15, IEEE Xplore Press, Tsukuba, pp: 489-492.

Kimia, B. and H.C. Aras, 2010. HINDSITE: A userinteractive framework for fragment assembly. Proceedings of the IEEE Computer Society Conference on Digital Object Identifier, Jun. 13-18, IEEE Xplore Press, San Francisco, CA, pp: 62-69. DOI: 10.1109/CVPRW.2010.5543532

Leitao, H.C.G. and J. Stolfi, 2005. Measuring the information content of fracture lines. Int. J. Comput. Vision, 65: 163-174. DOI: 10.1007/s1 1263-005-3226-8

Makridis, M. and P. Daras, 2012. Automatic classification of archaeological pottery sherds. ACM J. Comput. Cultural Heritage, 5: 1-21. DOI: $10.1145 / 2399180.2399183$

Oxholm, G. and K. Nishino, 2012. A flexible approach to reassembling thin artifacts of unknown geometry. J. Cultural Heritage, 14: 51-61. DOI: 10.1016/j.culher.2012.02.017

Skembris, A.S., C. Papaodysseus and E. Koukoutsis, 2012. 2D fragmented object reconstruction with the use of the chromatic and thematic content. J. Patt. Analysis Applic., 15: 133-146. DOI: 10.1007/s10044-011-0251-9

Smith, P., D. Bespalov, A. Shokoufandeh and P. Jeppson, 2010. Classification of archaeological ceramic fragments using texture and color descriptors. Proceedings of the IEEE Computer Vision and Pattern Recognition Workshop, Jun. 1318, IEEE Xplore Press, San Francisco, CA, pp: 4954. DOI: 10.1109/CVPRW.2010.5543523

Son, K., E.B. Almeida and D. Cooper, 2013. Axially symmetric 3D pots configuration system using axis of symmetry and break curve. Proceedings of the 2013 IEEE Conference on Computer Vision and Pattern Recognition, (VPR' 13), Washington, DC, USA, pp: 257-264. DOI: 10.1109/CVPR.2013.40

Sturmfels, B., 2002. Solving Systems of Polynomial Equations. 1st Edn., American Mathematical Soc., ISBN-10: 0821889419, pp: 152. 
Toler-Franklin, C., B. Brown, T. Weyrich, T. Funkhouser and S. Rusinkiewicz, 2010. Multifeature matching of fresco fragments. ACM Trans. Graphics, 29: 1-185. $\quad$ DOI: $10.1145 / 1882261.1866207$

Ying, Q.L. and W.K. Gang, 2010. Kernel fuzzy clustering based classification of ancient-ceramic fragments. Proceedings of the 2nd IEEE International Conference on Information Management and Engineering, Apr. 16-18, IEEE Xplore, Press, Chengdu, pp: 348-350. DOI: 10.1109/ICIME.2010.5477818
Zhang, H., Y. Wang and F. Zhang, 2011. Twodimensional contour matching algorithm based on multiscale space. Proceedings of the $3 \mathrm{rd}$ International Conference on Computer Research and Development, Mar. 11-13, IEEE Xplore Press, Shanghai, pp: 458-461. DOI: 10.1109/ICCRD.2011.5764057 